\title{
Extracción y caracterización de un ácido húmico, y su evaluación fisicoquímica de coagulación en presencia de $\mathrm{Fe}^{3+} \mathrm{y} \mathrm{Al}^{3+}$
}

\author{
Extraction and characterization of a humic acid, and its physicochemical evaluation of \\ coagulation in presence of $\mathrm{Fe}^{3+}$ and $\mathrm{Al}^{3+}$
}

\author{
Abril Villagrán-Manilla, Eduardo D. Ibarra-Coria, Alfredo Guevara-Lara, Silvia Nieto-Velázquez \\ Área Académica de Química, Universidad Autónoma del Estado de Hidalgo, Mineral de la Reforma, Hidalgo, 42184, \\ México
}

\begin{abstract}
:
In this research the extraction of a humic acid (HA) from a soil of Tolcayuca (Hidalgo State) were performed using $\mathrm{Na}_{2} \mathrm{P}_{2} \mathrm{O}_{7} .10 \mathrm{H}_{2} \mathrm{O}$ and $\mathrm{NaOH}$ as extracting agents. Physicochemical analysis of studied HAs (commercial CHEPAPEX HA-CH and extracted in the laboratory HA-To) showed polyacid and high chemical stability characteristics, which is corroborated with its ability to coagulate with the trivalent metals through zeta potential values. It was shown that there is a rapid interaction of HA-Metal in less than 5 min and especially when increasing the concentration of the metal $(1,2$ and $3 \mathrm{mM})$ at a $\mathrm{pH}$ of 5 , due to the deprotonation of carboxylic groups $(-\mathrm{COOH})$ principally, that can coordinate under these conditions, reducing the net negative charge and enabling such complexes, to approach one another and interact through hydrogen bonding.
\end{abstract}

Keywords:

Humic-acid, metal, interaction, coagulation, zeta potential.

\section{Resumen:}

En esta investigación se realizó la extracción de un ácido húmico (AH) de un suelo de Tolcayuca (estado de Hidalgo), empleando $\mathrm{Na}_{2} \mathrm{P}_{2} \mathrm{O}_{7} .10 \mathrm{H}_{2} \mathrm{O}$ e $\mathrm{NaOH}$ como agentes extractantes. El análisis fisicoquímico de los AHs en estudio (comercial CHEMAPEX AH$\mathrm{CH}$ y extraído en el laboratorio AH-To) mostraron características poliácidas y alta estabilidad química, lo cual se corrobora con su capacidad de coagulación con los metales trivalentes por medio de lecturas de potencial zeta. Los datos obtenidos evidenciaron que existe una rápida interacción $A H$-Metal en un tiempo menor a 5 min y sobre todo al incrementar la concentración del metal (1, 2 y 3 $\mathrm{mM}$ ) a un $\mathrm{pH}$ de 5, debido principalmente a la desprotonación de los grupos carboxílicos (-COOH), que bajo estas condiciones pueden coordinarse, reduciendo la carga neta negativa y permitiendo así que tales complejos se aproximen e interactúen a través de puentes de hidrógeno.

\section{Palabras Clave:}

Ácido-húmico, metal, interacción, coagulación, potencial zeta.

\section{Introducción}

La materia orgánica (MO) es un componente que se encuentra en un $5 \%$ en la capa arable (horizonte A) de un suelo y como sólidos suspendidos en aguas superficiales y subterráneas. Está
MO presente en agua, además de causar un mal aspecto físico en cuento a su color amarillento o café (agua sucia), causa problemas en el crecimiento microbiológico durante el tratamiento de la misma y en las redes de distribución, por otro lado, la MO juega un papel crítico en la formación de subproductos de desinfección, proporcionando material 
precursor para una gran variedad de compuestos altamente tóxicos, lo que hace que las interacciones entre la MO y ciertos compuestos sean un tema interesante para la investigación. Las estructuras de la MO son específicas para el tipo de agua y pueden contener grupos funcionales reactivos como fenol ($\mathrm{OH})$ y ácidos carboxílicos (-COOH) que pueden participar en reacciones de óxido-reducción o formar complejos. Hasta ahora, los efectos de la MO sobre la adsorción, la especiación y la movilidad con metales han sido investigados por varios autores [1-4]. Sin embargo, se sabe poco sobre las interacciones que presenta con ciertos compuestos, siendo necesaria una mayor investigación para un factor clave en la selección de las tecnologías de tratamiento del agua potable.

Es importante mencionar que en la MO encontramos diferentes sustancias como carbohidratos, proteínas, aminas, esteres, cetonas, entre otros, pero también encontramos a una serie de compuestos de elevado peso molecular llamados Sustancias Húmicas (SHs), también conocidas con el nombre de humus. Son la fracción más estable de la MO de los suelos y puede persistir por miles de años, se originan de la degradación microbiana de biomoléculas de plantas (y posiblemente también de animales), por ejemplo lignina, dispersados en el ambiente luego de la muerte de las células. La materia húmica es una estructura supramolecular de moléculas bio-orgánicas de tamaño relativamente pequeño que se autoensamblan principalmente mediante interacciones débiles (fuerzas de Van der Waals, $\pi-\pi$, e interacciones $\mathrm{CH}-\pi$ ), formando estructuras de gran tamaño y extremadamente complejas y confusas, donde posiblemente no hay dos macromoléculas exactamente iguales, debido a que su cantidad y composición va a depender de diferentes parámetros climatológicos y fisicoquímicos de la zona [5].

Las SHs pueden clasificarse en términos de sus propiedades solubles, es decir en ácidos húmicos (AHs) que son extraídos de los suelos con soluciones alcalinas y convertidos en precipitados insolubles en medio ácido; en ácidos fúlvicos (AFs) solubles tanto en soluciones alcalinas como ácidas (solubles en todo el intervalo del $\mathrm{pH}$ ) y en huminas que es la $\mathrm{MO}$ en los residuos alcalinos [6]. Es importante destacar que no existen límites definidos entre los AHs, AFs y las huminas. Todos ellos son parte de un sistema supramolecular extremadamente heterogéneo y las diferencias entre estas subdivisiones son debido a variaciones en la acidez, grado de hidrofobicidad (contenido de restos aromáticos y alquílicos de cadena larga) y la autoasociación de moléculas por efectos entrópicos. Sin embargo, en este trabajo de investigación nos enfocamos a los AHs, debido a que se les considera los componentes principales de la materia orgánica de suelo, agua, o sedimento, debido a la estabilidad química que presentan en relación con su estructura molecular que se compone de un esqueleto aromático (heteropolímero condensado) [7-8], con una gran cantidad y variedad de grupos funcionales, principalmente carboxílicos e hidróxidos (-COOH y -OH), que le confieren ciertas características químicas muy peculiares como una elevada capacidad de intercambio catiónico (CIC), son fuertes quelatos, reguladores de $\mathrm{pH}$ y agentes oxidantes $\mathrm{y} / \mathrm{o}$ reductores [1,9]. Por lo tanto, estos grupos funcionales confieren las propiedades ácidas, cuyo hidrógeno es susceptible a las reacciones de sustitución, es decir, la acidez o reacción de los AHs viene determinada en su mayor parte por la cantidad de cationes hidrógeno fijados en relación con los demás iones. No obstante, estos ácidos tienen gran capacidad de absorción para compuestos hidrofílicos e hidrofóbicos.

Por todo lo anteriormente expuesto, relacionado con todas las características fisicoquímicas y microbiológicas que presentan los AHs como compuestos abundantes en la MO, se pretende evaluar el efecto de coagulación aprovechando la gama de interacciones con diferentes metales. En este proceso sería la interacción con $\mathrm{Fe}^{3+}$ y $\mathrm{Al}^{3+}$ permitiendo la eliminación de $\mathrm{MO}$ en el tratamiento de agua y al mismo tiempo analizar los mecanismos de biodisponibilidad y transporte que presentan los metales en el suelo, mediante la formación de agregados o aglomerados.

Se inició con la extracción de un ácido húmico (AH-To) de muestra de suelo, posteriormente, se caracterizó por métodos espectroscópicos que revelaron un AH poliácido, corroborando estos resultados con la capacidad de coagulación al interaccionar con los iones trivalentes $\left(\mathrm{Fe}^{3+} \mathrm{y} \mathrm{Al}^{3+}\right)$ evaluada mediante lecturas de potencial zeta, evidenciando una rápida interacción del $A H$-metal e incrementando su coagulación al aumentar la concentración del catión a un pH de 5 [2-3, 10]. Los resultados se compararon con un AH comercial CHEMAPEX $(\mathrm{AH}-\mathrm{CH})$ caracterizado y evaluado en trabajos anteriores [11$12]$.

\section{Desarrollo experimental}

\section{Obtención de la muestra de suelo.}

En la obtención y preparación de la muestra de suelo $(\approx 1 \mathrm{~kg}$ de muestra) se realizó escarbando de $15-20 \mathrm{~cm}$ de profundidad de la capa arable (horizonte A) a partir de un lugar no contaminado en el estado de Hidalgo (Tolcayuca) y alejado de la civilización. No obstante, para la recolección de la muestra de suelo. Se realiza un análisis cualitativo de identificación principalmente con base al color café obscuro o negro (mayor cantidad de MO), en relación con lugares boscosos que presentan una constante degradación y transformación de la biomasa vegetal y animal [13-14]. A las muestras de suelo se les eliminó basura, piedras y raíces, posteriormente se secaron en un horno convencional a una temperatura de $60{ }^{\circ} \mathrm{C}$ (equipo LINDBERG, modelo GO1310A-1), se tamizaron hasta obtener un tamaño de partícula de $1.0 \mathrm{~mm}$ y se colocaron en recipientes completamente limpios y herméticos.

\section{Método de extracción de la muestra de AH-To.}

En la extracción y purificación del AH se utilizó el método de Kononova (1982), con ciertas modificaciones con respecto a la separación de los AHs por centrifugación [12-15]. Para la extracción del AH se pesaron $15 \mathrm{~g}$ de suelo y se transfirieron a un matraz Erlenmeyer con capacidad de $250 \mathrm{~mL}$. Posteriormente, se agregaron $120 \mathrm{~mL}$ de una solución recién preparada de $\mathrm{Na}_{2} \mathrm{P}_{2} \mathrm{O}_{7} .10 \mathrm{H}_{2} \mathrm{O}$ e $\mathrm{NaOH}$, (11.15 g de $\mathrm{Na}_{2} \mathrm{P}_{2} \mathrm{O}_{7}$ $.10 \mathrm{H}_{2} \mathrm{O}$ y $1 \mathrm{~g}$ de $\mathrm{NaOH}$ en $250 \mathrm{~mL}$ de solución) a $\mathrm{pH} \approx 13$; el matraz se cerró perfectamente con una septa para evitar la entrada de $\mathrm{CO}_{2}$ y se mantuvo en agitación durante $30 \mathrm{~min}$, se dejó reposar aproximadamente $24 \mathrm{~h}$. Finalmente se filtró utilizando papel filtro whatman No. 42. Al filtrado obtenido se le añadió aproximadamente $2.5 \mathrm{~mL}$ de $\mathrm{HCl}$ concentrado, se agitó cuidadosamente y se dejó reposar hasta observar la precipitación de AH. La mezcla se centrifugó por 10 min a 2300 rpm, desechando el sobrenadante y así continuando la operación 
hasta tener una parte mínima de líquido, prosiguiendo con el filtrado a vacío utilizando membranas Millipore de tipo GVHP $0.22 \mu \mathrm{m}$. El sólido se secó en la estufa a una temperatura de 60 ${ }^{\circ} \mathrm{C}$ durante 2 h aproximadamente; se retiró y se colocó en el desecador alrededor de $30 \mathrm{~min}$ y finalmente se pesó. Las muestras obtenidas se preservaron (recipientes color ámbar) para su posterior caracterización con diferentes métodos y técnicas analíticas.

Caracterización de los AHs (comercial: $\mathrm{AH}-\mathrm{CH}$ y extraído: $\mathrm{AH}-\mathrm{To})$

Se trabajó con dos AHs (AH-CH y AH-To), considerando que el AH-CH (comercial Chemapex Praha S.R.O.) fue caracterizado, evaluado y presentado en trabajos anteriores [1112]. No obstante, considerando los mismos métodos y técnicas analíticas para la caracterización del AH-To, a partir de espectroscopia UV-Vis (absorbancia, concentración, coeficiente de absortividad molar, aromaticidad, peso molecular promedio y cociente $\mathrm{E}_{4} / \mathrm{E}_{6}$ ) y por espectroscopia de IRTF (grupos funcionales principales: $-\mathrm{COOH},-\mathrm{C}=\mathrm{O},-\mathrm{OH}$, $\mathrm{CH}_{3}$, entre otros).

\section{Espectroscopia UV-Vis}

Las propiedades espectroscópicas como la absortividad molar $\left(\varepsilon_{280}\right)$, porcentaje de aromaticidad, peso molecular promedio y el grado de humificación de los AHs mediante el valor del cociente $\mathrm{E}_{4} / \mathrm{E}_{6}$. Se determinaron en un equipo Perkin Elmer modelo Lambda 40, en el cual se obtuvieron los espectros a longitudes de onda $\lambda=$ de 200 a $700 \mathrm{~nm}$ a partir de una solución de 10 ppm de AH (dilución de la solución madre de $1000 \mathrm{mg} / \mathrm{l}$ ) [16], registrando las absorbancias a diferentes longitudes de onda principalmente a 280, 465 y $665 \mathrm{~nm}$. Se determinó el coeficiente de absortividad molar $\left(\varepsilon_{280}\right)$ a $280 \mathrm{~nm}$ utilizando la ecuación de Lambert-Beer.

$$
\text { Absorbancia }=\varepsilon \text { c I }
$$

El Porcentaje de aromaticidad y el peso molecular promedio se calcularon utilizando las siguientes ecuaciones correlacionadas con la absortividad molar [17].

$$
\begin{aligned}
& \text { Aromaticidad }=0.05 \varepsilon_{(280)}+6.74 \\
& \text { P.M. }=3.99 \varepsilon_{(280)}+490
\end{aligned}
$$

Se calculó también la relación o el cociente $\mathrm{E}_{4} / \mathrm{E}_{6}$ (absorbancia de $465 \mathrm{~nm}$ dividida entre la absorbancia de $665 \mathrm{~nm}$ ), parámetro utilizado para correlacionar el grado de humificación.

\section{Espectroscopia de IRTF}

Se utilizó un espectrofotómetro Perkin Elmer modelo Spectrum GX, preparando pastillas con $2.0 \mathrm{mg}$ de muestra de húmico en $200 \mathrm{mg}$ de la sal binaria de $\mathrm{KBr}$ y utilizando el intervalo de número de onda comprendido de 4000 a $400 \mathrm{~cm}^{-1}$.

Proceso de coagulación de los AHs en presencia de $\mathrm{Fe}^{3+}$ y $\mathrm{Al}^{3+}$. Para llevar a cabo el proceso de coagulación de los AHs (AH$\mathrm{CH}$ y $\mathrm{AH}-\mathrm{To}$ ), al interaccionar con cada uno de los metales trivalentes fue necesario establecer la concentración de AH e ir variando la concentración del metal trivalente.

\section{Preparación de soluciones madre}

Se inició por preparar las soluciones madre de $1000 \mathrm{mg} / \mathrm{l}$ de cada uno de los AHs (10 mg de AH en $360 \mu \mathrm{L}$ de $\mathrm{NaOH} 1 \mathrm{~N}$ y se aforó con agua desionizada hasta $10 \mathrm{~mL}$ ) [16], solución madre de $100 \mathrm{mM}$ de $\mathrm{NaCl}$ como electrolito soporte y solución madre de $10 \mathrm{mM}$ de cada una de las sales de los metales en estudio $\left(\mathrm{FeCl}_{3}\right.$ y $\left.\mathrm{Al}\left(\mathrm{ClO}_{4}\right)_{3} .9 \mathrm{H}_{2} \mathrm{O}\right)$.

\section{Proceso de coagulación.}

Se prepararon cuatro soluciones, la primera solución se le agregó $50 \mathrm{mg} / \mathrm{l}$ de $\mathrm{AH}$ y $15 \mathrm{mM}$ de $\mathrm{NaCl}$ como electrolito soporte (solución blanco), con las otras tres soluciones se les agregó la misma cantidad de húmico y electrolito soporte pero se evaluaron diferentes concentraciones de la sal del metal trivalente a 1, 2 y $3 \mathrm{mM}$ ajustando todas las soluciones a $\mathrm{pH}$ de 5 , y posteriormente se realizó el mismo procedimiento descrito pero ahora ajustando las soluciones a $\mathrm{pH}$ de 7 . Se dejaron en reposo aproximadamente $16 \mathrm{~h} \quad \mathrm{y}$ se obtuvieron las correspondientes lecturas por potencial Zeta $(\zeta)$ utilizando un equipo Malvern modelo Zetasizer nano series [2-3].

\section{Resultados y Discusión}

El método de extracción utilizado resulto ser un tratamiento rápido, económico y eficiente comparado con otros métodos más laboriosos y sobre todo más drásticos en cuanto a los agentes extractantes [15], sin embargo, es importante mencionar que el método utilizado no es recomendable para suelos muy mineralizados o con bajas cantidades de MO [18].

\section{Caracterización de los AHs (AH-CH y AH-To)}

Espectroscopia UV-Vis. A pesar de que los espectros de los AHs caracterizados presentan la misma tendencia (Figura 1), existen pequeñas diferencias que arrojan en las absorbancias correspondientes con base a las diferentes longitudes de onda y aunado a ello en cuanto a diferentes valores en su respectivo cociente $\mathrm{E}_{4} / \mathrm{E}_{6}$ (Tabla I). No obstante, de acuerdo a lo reportado en la literatura [1], los AHs que se analizaron presentan valores del cociente $\mathrm{E}_{4} / \mathrm{E}_{6}<5$, lo cual indica que son ácidos maduros o humificados (mayor carácter aromático y peso molecular), decreciendo en el siguiente orden: AH-CH > AH-To.

Esto último se correlaciona y coincide con el porcentaje de aromaticidad y el peso molecular promedio obtenidos mediante el coeficiente de absortividad molar a partir de las ecuaciones 1,2 y 3 [15].

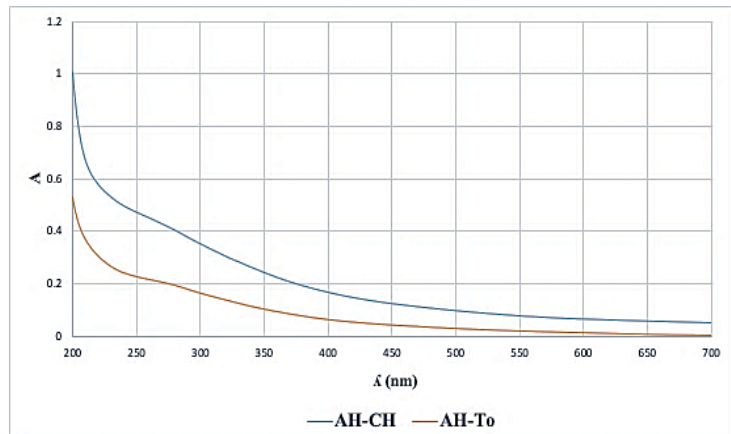

Figura 1. Espectros de absorción UV-Vis: $\mathrm{AH}-\mathrm{CH}$ y $\mathrm{AH}-\mathrm{To}$, $10 \mathrm{mg} / \mathrm{L}, \mathrm{pH}=11.09$, longitud de celda: $1 \mathrm{~cm}$ 
Los resultados del análisis por espectroscopia UV-Vis a partir de la absorbancia a $280 \mathrm{~nm}$ y de la concentración de carbono orgánico (CO) con base al AH, se presentan en la siguiente tabla:

Tabla I. Parámetros químicos de los AHs en estudio por espectroscopia UV-vis

\begin{tabular}{|ccc|}
\hline \multirow{2}{*}{ Parámetros } & \multicolumn{2}{c|}{ ÁCIDOS HÚMICOS } \\
\cline { 2 - 3 } & AH-CH & AH-To \\
\hline \hline A & 0.4039 & 0.1958 \\
$\varepsilon_{(280)} /(\mathrm{L}$ (mol de CO) & \\
$\left.1 \mathrm{~cm}^{-1}\right)$ & 1409.00 & 758.00 \\
\%Aromaticidad & 77.17 & 44.64 \\
P.M. & 6110.32 & 3514.42 \\
E4/E6 & 2.07 & 4.98 \\
\hline
\end{tabular}

Espectroscopia de IRTF. Los espectros de IRTF de los AHs (AH-CH y AH-To) revelaron la presencia de bandas de absorción muy similares, independientemente de su fuente u origen natural, aunque con pequeñas diferencias en la intensidad y desplazamientos de los picos. Esto permite proponer la presencia de los mismos grupos funcionales principales y más abundantes del tipo: $-\mathrm{OH},-\mathrm{COOH},-\mathrm{C}=\mathrm{O}$, de alcoholes, fenoles, ésteres, cetona o amidas, entre otros, aunque en diferente proporción (Figura 2).
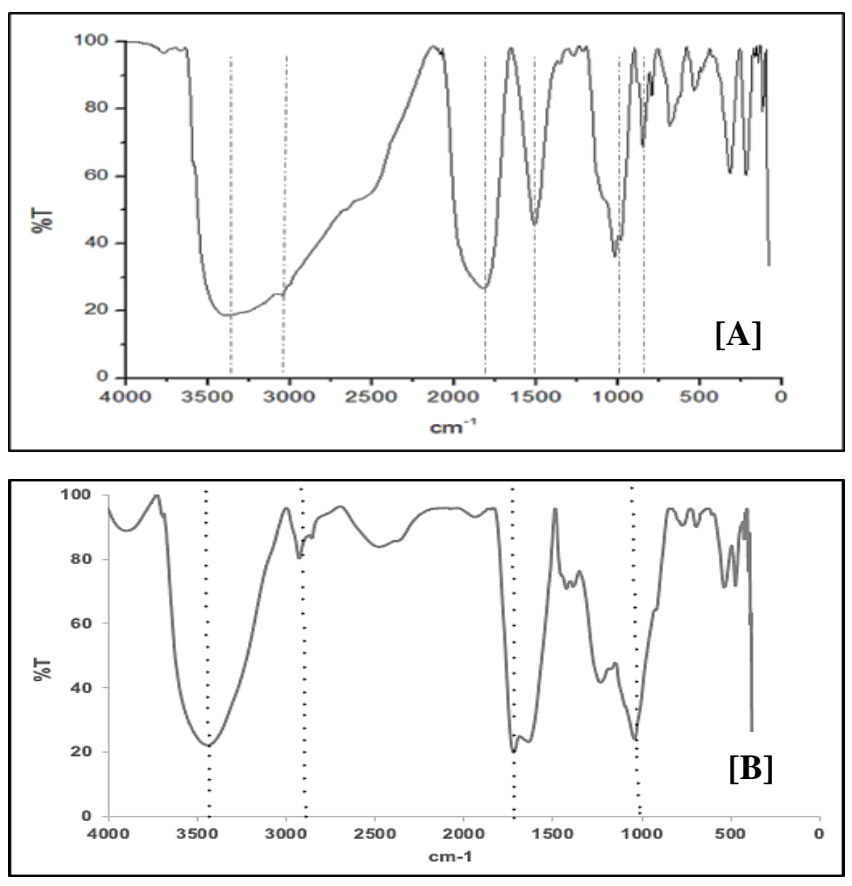

Figura 2. Espectro de IRTF: [A] AH-CH y [B] AH-To, analizados en pastilla: con $2.0 \mathrm{mg}$ demuestra en $200 \mathrm{mg}$ de $\mathrm{KBr}$
En los espectros de IRTF de la Figura 2 se observa la presencia de bandas de absorción que caracterizan las vibraciones de alargamiento y de flexión dentro y fuera del plano, así como las huellas dactilares (912 a $450 \mathrm{~cm}^{-1}$ ) en las diferentes agrupaciones atómicas (Tabla II) [19].

Tabla II. Asignación de las bandas de absorción de los espectros de infrarrojo de la Figura 2

\begin{tabular}{|c|c|}
\hline$v\left(\mathrm{~cm}^{-1}\right)$ & Asignación \\
\hline $3650-3100$ & $\mathrm{VO}-\mathrm{H}, \mathrm{N}-\mathrm{H}$ \\
\hline $3100-2840$ & VC-H \\
\hline 1710-1585 & $V_{C}=\mathrm{O}, \mathrm{C}=\mathrm{C}$ \\
\hline $1440-1330$ & $\begin{array}{c}\int \mathrm{O}-\mathrm{H},=\mathrm{C}-\mathrm{H} \\
\text { vC-N }\end{array}$ \\
\hline $1320-1100$ & $\begin{array}{l}\mathrm{V} C-\mathrm{O} \\
\int_{\mathrm{C}} \mathrm{C}=\mathrm{O}\end{array}$ \\
\hline $1200-1020$ & VC-C \\
\hline $912-450$ & huellas dactilares \\
\hline
\end{tabular}

Una vez caracterizadas las señales principales en los espectros, es importante señalar las diferencias más representativas de los AHs: El AH-CH (Figura 2-A), por su mayor peso molecular y aunado a ello la cantidad de grupos funcionales y la presencia de humedad, se obtiene una señal muy ancha y no se logra observar la señal de las cadenas alifáticas $\left(2970-2850 \mathrm{~cm}^{-1} \mathrm{C}-\mathrm{H}\right.$ alcanos y $\mathrm{CH}_{3}$ ), no obstante, el espectro nos presenta otras dos señales importantes a parte de la señal del carbonilo, es la banda de los anillos aromáticos $\left(1600-1500 \mathrm{~cm}^{-1} \quad \mathrm{C}=\mathrm{C}\right)$ y de los alcoholes y ácidos carboxílicos (1300-1050 $\mathrm{cm}^{-1}$ de C-O). El AH-To (Figura 2-B) se observa una señal muy definida de la banda característica de los grupos funcionales: $-\mathrm{COOH}$ y $-\mathrm{OH}$ (3650-3100 $\mathrm{cm}^{-1} \mathrm{O}-\mathrm{H}$ ) y comprobando el aumento de grupos $\mathrm{COOH}$ aparece un ensanchamiento de la banda característica del grupo carbonilo $-\mathrm{C}=\mathrm{O}\left(1710-1730 \mathrm{~cm}^{-1}\right)$, nuevamente confirmando la acidez de este ácido, por otro lado, presenta una disminución de la señal de cadenas alifáticas (2943-2850 cm $\mathrm{C}-\mathrm{H}$ alcanos y $\mathrm{CH}_{3}$ ) y un aumento de la señal $\mathrm{C}-\mathrm{O}$ de los alcoholes y ácidos carboxílicos (1300-1050 $\mathrm{cm}^{-1}$ de C-O) lo que se relaciona con su acidez [20].

De esta forma se puede concluir que en los dos AHs, la aromaticidad, peso molecular y el grado de humificación es mayor para el AH-CH en comparación con el AH-To por espectroscopia UV-Vis. Sin embargo, en lo que se refiere a la acidez con base en la señal característica de grupos funcionales carboxílicos (-COOH) identificados en los espectros de IRTF parece que es mayor para el AH-To en comparación con el AH$\mathrm{CH}$, sería cuestión de corroborar mediante métodos clásicos por vía húmeda (valoraciones acido-base).

Estudio del proceso de coagulación de los AHs en presencia de $\mathrm{Fe}^{3+} \mathrm{oAl} \mathrm{l}^{3+}$

El efecto positivo sobre el $\zeta$ (cuando el potencial se vuelve menos negativo) debido la presencia del metal (contra-ion), se explica por la reducción de la carga del AH que se unen a los grupos carboxílicos y/o fenólicos negativamente cargados. La magnitud de esta diferencia puede relacionarse también con la afinidad entre la macromolécula y los iones metálicos, en este caso con el correspondiente metal trivalente, quienes presentan 
una mayor interacción con los grupos carboxílicos (-COO), tal y como se indica en literatura [2]. Así, es posible ver que los cambios en el valor del $\zeta$ no sólo es el efecto de la modificación en la fuerza iónica de la disolución y, por lo tanto, la compresión de la doble capa que rodea a la macromolécula, sino una medida de la atracción entre AHs y diferentes compuestos iónicos.

Debido a la mínima influencia que tiene la sal de $\mathrm{Na}^{+}$en potencial zeta del húmico, y con la finalidad de aumentar la conductividad eléctrica de la disolución, a los posteriores análisis se realizaron añadiendo una concentración $15 \mathrm{mM}$ de $\mathrm{NaCl}$ como electrolito soporte. Así, se evaluó bajo estas condiciones el efecto del incremento de la concentración del metal a 1, 2 y 3 mM, a valores de pH 5 y 7 y después de un tiempo de 16 h de reacción. Los resultados se observan en las siguientes figuras:

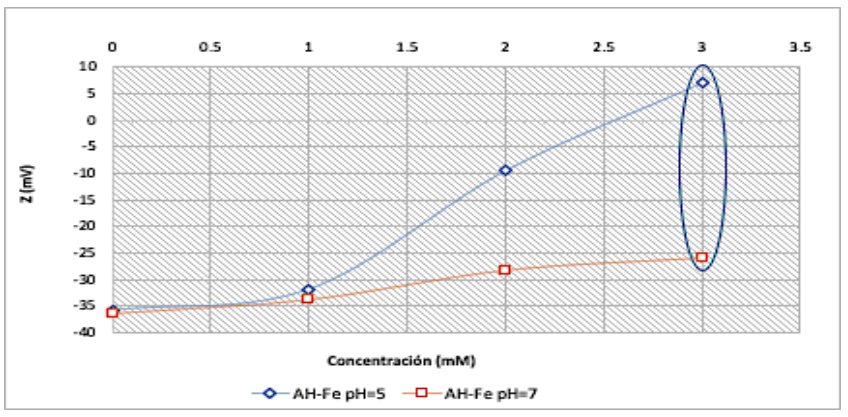

Figura 3. Efecto de la concentración de $\mathrm{Fe}^{3+}$ en el $\zeta$ del AH$\mathrm{CH}$ a $\mathrm{pH}=5$ y 7

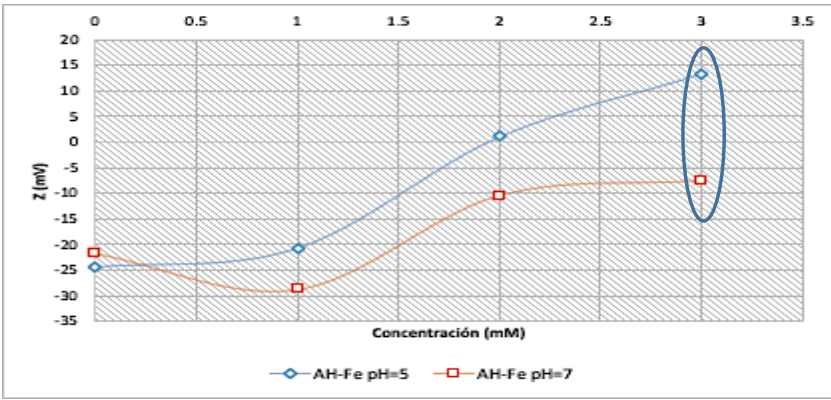

Figura 4. Efecto de la concentración de $\mathrm{Fe}^{3+}$ en el $\zeta$ del AHTo a $\mathrm{pH}=5$ y 7

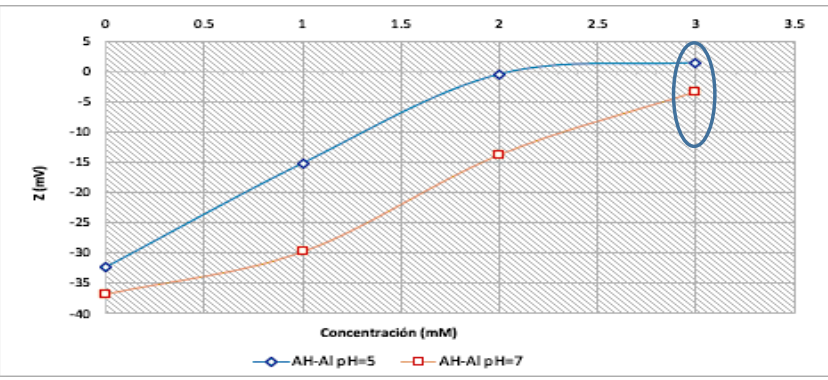

Figura 5. Efecto de la concentración de $\mathrm{Al}^{3+}$ en el $\zeta$ del AH$\mathrm{CH}$ a $\mathrm{pH}=5$ y 7

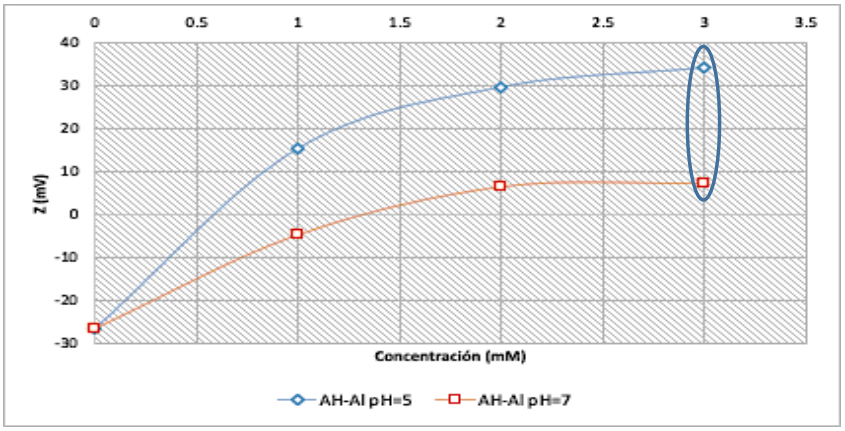

Figura 6. Efecto de la concentración de $\mathrm{Al}^{3+}$ en el $\zeta$ del AH-To a $\mathrm{pH}=5$ y 7

En la Tabla III se presentan los valores promedio obtenidos del $\zeta$ de los AHs con electrolito soporte en ausencia del metal y podemos observar que su $\zeta$ es independiente del $\mathrm{pH}$. Sin embargo, el efecto de la variación de la concentración (1, 2 y 3 $\mathrm{mM}$ ) del metal trivalente y del $\mathrm{pH}$ (5 y 7), mostró que los valores promedio del $\zeta$ de los mismos AHs si influye y la interacción AH-Metal aumenta a mayor concentración y a un $\mathrm{pH}$ de 5, se observa como los resultados se vuelven menos negativos o positivos (Tabla IV).

Tabla III. Determinación del $\zeta$ de los AHs $(50 \mathrm{mg} / \mathrm{l})$ a $\mathrm{pH}=5$ y 7

\begin{tabular}{|c|c|c|}
\hline \multirow{2}{*}{ Muestra } & \multicolumn{2}{|c|}{ Potencial Zeta $(\zeta)$} \\
\hline & $\mathrm{pH}=5$ & $\mathbf{p H}=7$ \\
\hline AH-CH & $-34.1(2.45)$ & $-36.6(0.19)$ \\
\hline AH-To & $-25.4(1.41)$ & $-24.1(3.30)$ \\
\hline
\end{tabular}

(en paréntesis \%DER de tres análisis)

Tabla IV. Determinación del $\zeta$ de los AHs $(50 \mathrm{mg} / \mathrm{l})$ con $\mathrm{Fe}^{3+}$ o $\mathrm{Al}^{3+}(1,2$ y $3 \mathrm{mM})$ a $\mathrm{pH}=5$ y 7

\begin{tabular}{|c|c|c|c|}
\hline \multirow{3}{*}{ Muestras } & \multicolumn{3}{|c|}{ 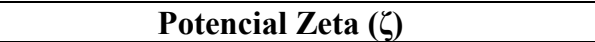 } \\
\hline & \multicolumn{3}{|c|}{$\mathrm{pH}=5$} \\
\hline & $1 \mathrm{mM}$ & $2 \mathrm{mM}$ & $3 \mathrm{mM}$ \\
\hline AH-CH-Fe & $-31.8(2.13)$ & $-9.53(7.30)$ & $7.03(7.16)$ \\
\hline AH-To-Fe & $-20.7(5.40)$ & $1.07(10.68)$ & $13.4(5.15)$ \\
\hline AH-CH-Al & $-15.2(1.38)$ & $-0.55(2.63)$ & $1.41(2.55)$ \\
\hline AH-To-Al & $15.4(4.59)$ & $29.7(4.30)$ & 34.2 \\
\hline Muestras & \multicolumn{3}{|c|}{$\mathrm{pH}=7$} \\
\hline AH-CH-Fe & $-33.8(5.37)$ & $-28.2(5.08)$ & $-25.8(2.75)$ \\
\hline AH-To-Fe & $-28.8(2.83)$ & $-10.5(6.87)$ & $-7.52(6.06)$ \\
\hline AH-CH-AI & $-29.8(4.67)$ & -13.9 & $-3.52(2.75)$ \\
\hline AH-To-Al & $-4.70(1.93)$ & $6.44(1.50)$ & 7.34 \\
\hline
\end{tabular}
$\leq 1$

Como ya se mencionó, el $\zeta$ se vuelve menos negativo o positivo cuando AH interacciona con el metal, lo cual muestra un proceso de coagulación que se ve favorecido con el AH-To (extraído en el laboratorio). Si correlacionamos estos resultados con la señal característica y definida de los ácidos carboxílicos $(-\mathrm{COOH})$ en el espectro de IRTF podemos de alguna forma precisar que este húmico presenta mayor capacidad de interacción o capacidad de intercambio catiónico. 


\section{Conclusiones}

Con el método de extracción utilizando, con $\mathrm{Na}_{2} \mathrm{P}_{2} \mathrm{O}_{7} \cdot 10 \mathrm{H}_{2} \mathrm{O}$ e $\mathrm{NaOH}$ como agentes extractantes y mediante la caracterización con los métodos espectroscópicos se obtuvo un AH-To poliácido de bajo peso molecular promedio, aromaticidad y humificación en comparación con el AH comercial (AH-CH), sin embargo, ambos AHs tienen una gran capacidad de interacción con $\mathrm{Fe}^{3+}$ y $\mathrm{Al}^{3+}$ al incrementar la concentración del metal, llevándose a cabo el proceso de coagulación y favoreciendo dicho proceso principalmente a un $\mathrm{pH}$ de 5 , provocando la disminución de la carga neta negativa de la macromolécula y permitiendo así que tales complejos se aproximen e interaccionen formando agregados o aglomerados y logrando la precipitación de los AHs como MO.

\section{Agradecimientos}

Silvia Nieto Velázquez agradece el financiamiento PRODEP, convenio no. 511-6/18-8644.

\section{Conflicto de intereses}

Los autores declaran que no existen conflictos de intereses.

\section{Referencias}

[1] Stevenson FJ. Humus Chemistry: Genesis, Composition and Reactions. $2^{\text {nd }}$ Ed. Willey Interscience, New York, NY. 1994.

[2] Kloster N, Brigante M, Zanini G, Avena M. Aggregation kinetics of humic acids in the presence of calcium ions. Colloids and Surfaces A: Physicochem. Eng. Aspects. 2013; 427: 76 - 82.

[3] Watson M.A, Tubi'c A, Agbaba J, Niki'c J, Maletíc S, Molnar Jazi'c J, Dalmacija B. Response surface methodology investigation into the interactions between arsenic and humic acid in water during the coagulation process. J. Hazardous Materials. 2016; 312: 150 -158.

[4] Ma J, Guo H, Weng L, Li Y, Lei M, Chen Y. Distinct effect of humic acid on ferrihydrite colloid-facilitated transport of arsenic in saturated media at different pH. Chemosphere. 2018; 212: 794-801.

[5] Aguilera-Herrero M. Tratado de edafología de México. $1^{\text {a }}$ Ed. México, D.F.: Facultad de Ciencias. 1989

[6] http://www.redalyc.org/pdf/339/33909608.pdfAlmendros G. Revisión analítica de sustancias húmicas en suelos y compost. soilACE. 2008: 239-255. Recuperado el 2 de mayo de 2018 de: https://www.soilace.com/pdf/pon2008/d26/Cas/04_GAlmendros.pdf.

[7] Schnitzer M. Soil Organic Matter. Soil Sci. 1991; 151: 41 - 58.

[8] Schulten H.R, Hempfling R. Influence of agricultural and management on humus compositon and dynamics: Classical and modern analytical techniques. Plant Soil. 1992; 142: 259 - 271.

[9] Plaza C, Brunetti G, Senesi N, Polo A. Molecular and quantitative analysis of metal ion binding to humic acids from sewage sludge and sludge-amended soils by fluorescence spectroscopy. Environ. Sci. Technol. 2006; 40(3): 917 - 923.

[10] Nieto-Velázquez S. Evaluación del Efecto de las Cinéticas de Agregación de los Ácidos Húmicos (AHs) en Presencia de $\mathrm{Na}^{+}, \mathrm{Ca}^{2+}$ y $\mathrm{Mg}^{2+}$. Revista Tendencias en Docencia e Investigación en Química. 2017; 3: 154 - 162 .

[11] Nieto-Velázquez S. Estabilidad de los ácidos húmicos y su influencia en la interacción con Cu(II) y Pb(II). Tesis de Doctorado, Universidad Autónoma del Estado de Hidalgo, México. 2010.

[12] Carpio-Domínguez K. Análisis fisicoquímico de ácidos húmicos y evaluación cinética de su agregación con iones $\mathrm{Na}^{+}, \mathrm{Ca}^{2+} \mathrm{y} \mathrm{Mg}^{2+}$. Tesis de Licenciatura, Universidad Autónoma del Estado de Hidalgo, México. 2017.
[13] Bohn HL, McNeal BL, O'connor GA. Química del suelo. $1^{\text {a }}$ Ed. México: Limusa. 1993.

[14] Ortega-Torres D. Química de suelos. Departamento de suelos. Universidad Autónoma de Chapingo. Texcoco, México. 1981.

[15] Kononova MM. Materia Orgánica del Suelo. $1^{\mathrm{a}}$ Ed. Oikos-tau, Barcelona, España; 1982: 63-105.

[16] Pacheco ML. Capillary Electrophoresis and MALDI-TOF Mass Spectrometry of Humic Acids. Tesis de Doctorado, Departamento de Química Analítica, Facultad de Ciencias, Universidad de Masaryk, República Checa. 2002.

[17] Chin YP, Alken G, Loughlin EO. (1994). Molecular weight, polydispersity, and spectroscopic properties of aquatic humic substances. Environ. Sci. Technol. 1994; (28): 1853 - 1858.

[18] Licona-Sánchez TJ. Extracción y Análisis Fisicoquímico de Ácidos Húmicos y su Interacción con Interacción con $\mathrm{Cu}(\mathrm{II})$ y $\mathrm{Pb}(\mathrm{II})$. Tesis de Licenciatura, Universidad Autónoma del Estado de Hidalgo, México. 2007.

[19] Tipping E. Cation binding by humic substances. West Nyack, N.Y. USA: Cambridge University Press. 2002.

[20] Rodríguez W, García PA, Fajardo A. Aplicaciones de técnicas espectroscópicas para el análisis de suelos. Universidad Militar Nueva Granada. 2016; 12(2): 228-251. 